\title{
VARIABILIDAD INTEROBSERVADOR EN EL DIAGNÓSTICO Y CLASIFICACIÓN DE LA RETINOPATÍA DIABÉTICA MEDIANTE BIOMICROSCOPÍA
}

\section{INTER-OBSERVER VARIABILITY IN THE DIAGNOSIS AND CLASSIFICATION OF DIABETIC RETINOPATHY THROUGH BIOMICROSCOPY}

\author{
MOLINA-FERNÁNDEZ E ${ }^{1}$, VALERO-MOLL MS ${ }^{2}$, PEDREGAL-GONZÁLEZ M \\ DÍAZ-RODRÍGUEZ E ${ }^{2}$, SÁNCHEZ-RAMOS JL ${ }^{1}$, SORIANO-VILLEGAS JM ${ }^{2}$
}

\section{RESUMEN}

Objetivo: Análisis de la variabilidad interobservador de la biomicroscopía utilizada para el diagnóstico de retinopatía diabética.

Métodos: Diseño: Observacional descriptivo. Valoración en paralelo, de forma ciega para los observadores, del grado de retinopatía diabética mediante biomicroscopía en pacientes diabéticos tipo 2 . Muestra: Para la evaluación del índice Kappa, con una estimación de una proporción de desacuerdo del 15\%, (precisión del 5\% intervalo de confianza del 95\%) muestra $\mathrm{n}=196$, (siendo «n» el número de ojos). Variables a medir: grado de retinopatía diabética, según la clasificación del ETDRS modificada. Resultados: La edad media de los pacientes fotografiados fue de 65,42 años (DE=9,91). De las 217 biomicroscopías realizadas, en 191 se encontró concordancia total. En 24 la discordancia fue tan sólo en un grado de la clasificación del ETDRS y en 2 la discordancia fue en dos grados. En ningún caso fue mayor. Kappa ponderado cuadrático $=0,876$,

\section{ABSTRACT}

Objective: Analysis of the inter-observer variability of biomicroscopy used for the diagnosis of Diabetic Retinopathy.

Methods: This was a descriptive study. Parallel observer-blind evaluations of the degree of retinopathy in type 2 diabetic patients, as defined on biomicroscopic photographs, were performed by two ophthalmologists. The sample size required for the Kappa index among ophthalmologists with a disagreement ratio of $15 \%$, precision ratio of $5 \%$ and confidence level of $95 \%$ is $n=196$ ( $« n$ » being the number of eyes). The only variable measured was the degree of Diabetic Retinopathy, according to the modified Early Treatment Diabetic Research Study (ETDRS) classification.

Results: The average age of the 217 patients photographed was of 65.42 years ( $\mathrm{SE}=9.91)$. In 191 instances there was total agreement between the 2 ophthalmologists. In 24 instances the discrepancy was only of one degree of the classification of the ETDRS,

\footnotetext{
Recibido: 23/1/07. Aceptado: 29/11/07. Salud. Hueva. España.

1 Doctor en Medicina.

2 Licenciado en Medicina.

Proyecto subvencionado FIS 01/0708 del Ministerio de Sanidad.

Correspondencia:

Eduardo Molina Fernández

Unidad Docente de Medicina Familiar y Comunitaria de Huelva

Hospital Vázquez Díaz, 4. a planta

Ronda Norte, s/n

21005 Huelva

España

E-mail: udhuelva@tiscali.es
}

Servicio de Oftalmología y Unidad Docente de Medicina Familiar y Comunitaria de Huelva. Hospital Juan Ramón Jiménez. Servicio Andaluz de 
IC95\%: 0,655-0,952 y Kappa ponderado lineal = 0,804, IC95\%: 0,729-0,878.

Conclusiones: El índice Kappa ponderado demuestra un grado de acuerdo «muy bueno». Las discrepancias producidas además de escasas no tienen trascendencia clínica, ya que no afectan a la decisión de tratamiento. La biomicroscopía leída por un solo oftalmólogo es un instrumento fiable como patrón de referencia para el diagnóstico de la retinopatía diabética.

Palabras clave: Diabetes mellitus tipo 2, retinopatía diabética, técnicas diagnósticas oftalmológicas, estándares de referencia, estudios de evaluación.

\section{INTRODUCCIÓN}

La Diabetes Mellitus tipo 2 (DM2) es un problema de salud que cada vez adquiere mayor relevancia. En España la prevalencia en mayores de 30 años oscila entre el 6,4 y el 10,3\%, encontrándose sin diagnosticar previamente del 3,5 al 5,9\% (1-3).

La Retinopatía Diabética (RD) es una de las complicaciones más importantes de la DM2, se han descrito cifras de prevalencia de (RD) muy variables, desde el 15 al $50 \%$, e incluso superiores dependiendo del tiempo de evolución de la diabetes $(4,5)$.

La población diabética presenta un riesgo relativo de pérdida de visión 20 veces superior al de la población no diabética (6). La RD causa el 70-80\% de los casos de ceguera por diabetes, convirtiéndose en la principal causa de déficit visual y ceguera en adultos de 20 a 74 años, presentándose en aproximadamente un 5\% de los pacientes con DM2 a los 10 años del diagnóstico (7).

La detección precoz (cribado de RD) tiene mucha importancia, ya que cuando aparece pérdida de agudeza visual acostumbra a ser demasiado tarde para que pueda realizarse un tratamiento eficaz. El tratamiento sería la fotocoagulación con láser que evita o retrasa la pérdida de visión en un buen número de pacientes con RD no proliferativa severa y $\mathrm{RD}$ proliferativa (8).

Por todo ello la recomendación general más extendida es de una exploración anual a todo paciente diabético, pudiéndose alargar este periodo si no hay factores de riesgo o lesión (9). Esto podría and in 2 the discrepancy was of two degrees. In no case was it greater than this. (Quadratic weighed Kappa $=0.876$, IC95\%: 0.655-0.952 and linear weighed Kappa $=0.804$, IC95\%: 0.729-0.878).

Conclusions: The Weighed Kappa index demonstrated a «very good» agreement of the degree of diabetic retinopathy. The discrepancies were slight, were of no clinical importance, and would not have affected treatment decisions. The results indicate that this examination, performed by a single ophthalmologist, can be utilised as a reference standard in Diabetic Retinopathy diagnosis (Arch Soc Esp Oftalmol 2008; 83: 23-28).

Key words: Diabetes mellitus type 2, diabetic retinopathy, diagnostic techniques ophthalmological, reference standard, evaluation studies.

provocar una tremenda sobrecarga asistencial en las unidades especializadas en diabetes ocular, lo que implicaría la existencia de pacientes con formas graves en lista de espera. La manera más eficaz de realizar el cribado de la RD es obtener imágenes de la retina para su clasificación, por lo que hay muchos estudios que tratan de validar esta técnica, comparándola con la biomicroscopía hecha por oftalmólogos (10-17), o por ópticos (18).

Sin embargo no hay estudios que hayan evaluado la variabilidad interobservador de la biomicroscopía, pese a que esta exploración hecha por un solo oftalmólogo, es la que se utiliza como patrón estándar de referencia.

Nuestro estudio trata de responder a esta incertidumbre.

\section{SUJETOS, MATERIAL Y MÉTODOS}

\section{Diseño}

Observacional descriptivo. Valoración en paralelo, de forma ciega para los observadores, del grado de retinopatía diabética mediante biomicroscopía.

\section{Sujetos de estudio}

Pacientes diabéticos tipo 2. Se excluyeron los pacientes diabéticos tipo 1 y los que hubiesen recibido tratamiento previo de fotocoagulación con láser. 


\section{Muestra, muestreo y marco del estudio}

Se trataba básicamente de evaluar el índice Kappa (19). Considerando el problema como la estimación de una proporción de desacuerdo, asumiendo que ésta se encuentra alrededor del $15 \%$, se podría obtener una precisión del $5 \%$ para un intervalo de confianza del $95 \%$ con una muestra de tamaño $\mathrm{n}=196$, (siendo «n» el número de ojos y no el de pacientes diabéticos) (20).

La muestra estaba formada por 217 ojos de pacientes con DM 2, seleccionados de forma consecutiva al acudir a consulta en el centro de salud y remitidos al servicio de oftalmología de nuestro centro, ambos de Huelva capital.

\section{Variables a medir}

Grado de retinopatía diabética, según la clasificación del ETDRS modificada (21).

\section{Consentimiento informado}

El protocolo de estudio fue aprobado por la comisión de investigación. A las personas seleccionadas para el mismo se les informó verbalmente y por escrito de su contenido, y firmaron su consentimiento.

\section{Recogida de datos y técnicas de medida de las variables}

A los pacientes dos oftalmólogos de forma ciega, les realizaron la biomicroscopía en el mismo día. Las variables a estudio y datos de filiación las recogieron de forma independiente cada uno en una hoja elaborada para tal fin.

La exploración se realizó con la lámpara de hendidura y una lente de no-contacto de 66 dioptrías (VOLK Super 66) ${ }^{\circledR}$. Se empleó la lente de contacto (Ocular MAINSTER Standard Focal/Grid) ${ }^{\circledR}$, si la visión no era adecuada.

Se recogieron también datos relativos a la transparencia de medios: cataratas $(\leq$ grado2), leucomas corneales y opacidades vítreas.

Análisis de los datos: Se utilizó el índice kappa ponderado lineal y cuadrático, para evaluar la concordancia en la lectura de la biomicroscopía de los oftalmólogos, ya que permite asignar distintos pesos según el grado de discordancia $(22,23)$.

Para la valoración de la fuerza de la concordancia del índice Kappa se utilizó la escala propuesta por Landis y Koch (24) (tabla I).

Programas de análisis estadístico utilizados: Los programas utilizados en este trabajo han sido la base de datos Access ${ }^{\circledR}$ para la recogida de datos, el programa estadístico SPSS ${ }^{\circledR}$ (SPSS for Windows, SPSS Inc, Chicago, USA) y la hoja de cálculo Excel $^{\circledR}$ (Excel for Windows, Illinois, USA) para el cálculo del índice Kappa.

\section{RESULTADOS}

La edad media de los pacientes fotografiados fue de 65,42 años (DE=9,91), con un mínimo de 35 y un máximo de 89 (fig. 1).

En cinco ojos no se pudo realizar la exploración (ambos oftalmólogos los consideraron no explorables), uno por enucleación, dos por cataratas muy

Tabla I. Concordancia del índice Kappa

\begin{tabular}{cc}
\hline Valor de $\mathrm{K}$ & Fuerza de la concordancia \\
\hline$<0,20$ & Pobre \\
$0,21-0,40$ & Débil \\
$0,41-0,60$ & Moderada \\
$0,61-0,80$ & Buena \\
$0,81-1$ & Muy buena \\
\hline \hline
\end{tabular}

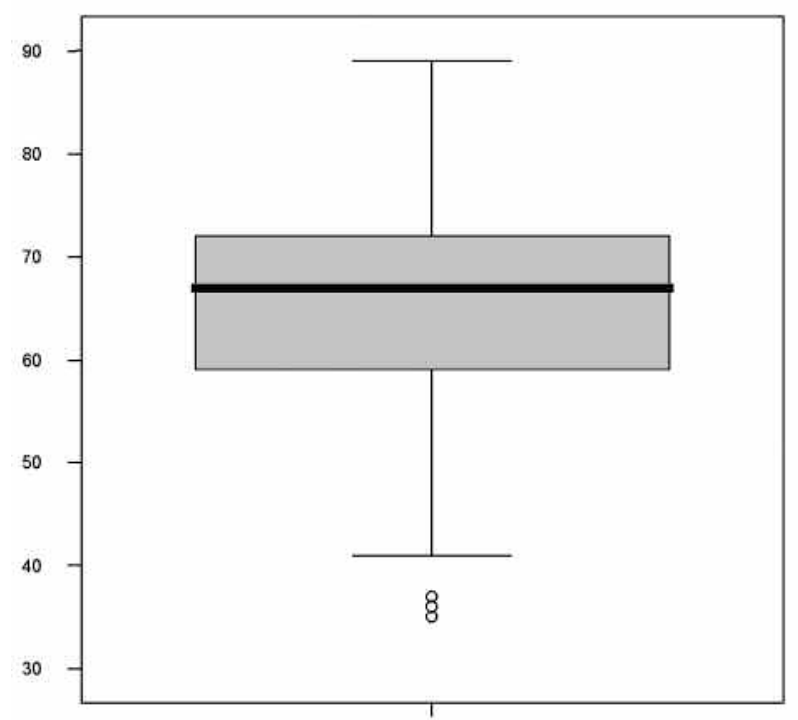

Fig. 1: Edad en años de los pacientes fotografiados. 
evolucionadas, otro por desprendimiento de retina crónico y en el quinto no constaba el motivo.

De los 217 ojos a los que se realizó la biomicroscopía, en 191 se encontró concordancia total entre los dos oftalmólogos, siendo etiquetados 151 de no RD, 16 de RD leve (RDL), 22 de RD moderada (RDM) y dos de RD severa (RDS). En 24 la discordancia fue tan sólo en un grado de la clasificación del ETDRS, en 17 era entre NO RD y RDL, en cinco entre RDL y RDM, uno entre RDM y RDS y uno entre RDS y retinopatía diabética muy severa (RDMS). En dos la discordancia fue en dos grados de la clasificación, entre NO RD y RDM. En ningún caso la discordancia fue mayor de dos niveles (tabla II).

Con estos resultados y la asignación de pesos según se establece en la tabla III, se calculó la concordancia global entre oftalmólogos, Kappa ponderado lineal $=0,804$, IC95\%: 0,729-0,878.

Con los mismos resultados y la asignación de pesos según se establece en la tabla IV, se calculó la concordancia global entre oftalmólogos, Kappa ponderado cuadrático $=0,876$, IC95\%: 0,655-0,952

En nueve de los 26 ojos con discordancias se encontraron problemas de transparencia de medios $(34,62 \%)$, frente a 42 en los 191 concordantes $(21,99 \%)$. Diferencia no estadísticamente significativa $\mathrm{P}=0,1542$.

Tabla II. Concordancia de la biomicroscopía entre dos oftalmólogoss, en los grados de retinopatía diabética

\begin{tabular}{lrrrrrr}
\hline & NO RD & RDL & RDM & RDS & RDMS & \\
\hline NO RD & 151 & 7 & 2 & & & 160 \\
RDL & 10 & 16 & 4 & & & 30 \\
RDM & & 1 & 22 & & & 23 \\
RDS & & & 1 & 2 & 1 & 4 \\
RDMS & 161 & 24 & 29 & 2 & 1 & 217 \\
& 161 &
\end{tabular}

No retinopatía diabética (NO RD), leve (RDL), moderada (RDM), severa (RDS) y muy severa (RDMS).

Tabla III. Asignación de pesos para calcular el Kappa ponderado lineal

\begin{tabular}{llllll}
\hline & NO RD & RDL & RDM & RDS & RDMS \\
\hline NO RD & 1 & 0,75 & 0,5 & 0,25 & 0 \\
RDL & 0,75 & 1 & 0,75 & 0,5 & 0,25 \\
RDM & 0,5 & 0,75 & 1 & 0,75 & 0,5 \\
RDS & 0,25 & 0,5 & 0,75 & 1 & 0,75 \\
RDMS & 0 & 0,25 & 0,5 & 0,75 & 1 \\
\hline \hline
\end{tabular}

Tabla IV. Asignación de pesos para calcular el Kappa ponderado cuadrático

\begin{tabular}{llllll}
\hline & NO RD & RDL & RDM & RDS & RDMS \\
\hline NO RD & 1 & 0,9375 & 0,75 & 0,4375 & 0 \\
RDL & 0,9375 & 1 & 0,9375 & 0,75 & 0,4375 \\
RDM & 0,75 & 0,9375 & 1 & 0,9375 & 0,75 \\
RDS & 0,4375 & 0,75 & 0,9375 & 1 & 0,9375 \\
RDMS & 0 & 0,4375 & 0,75 & 0,9375 & 1 \\
\hline \hline
\end{tabular}

\section{DISCUSIÓN}

Se calculó el Kappa ponderado con asignación de pesos lineales y cuadráticos. Existe en la literatura controversia sobre cual de estos es el más adecuado. Cuando se asignan pesos cuadráticos el valor del Kappa tiende a incrementarse de forma proporcional al número de categorías (25). En el caso de la RD consideramos que este último tiene más valor ya que asigna mayor diferencia en los pesos cuanto más alejadas estén las categorías en las discordancias, lo que se corresponde mejor con la trascendencia clínica de la prueba: no es lo mismo equivocarse en un grado de retinopatía, por ejemplo entre que no haya retinopatía o que sea leve, que confundir ausencia de retinopatía con una severa. El error del primer caso se subsanaría sin problemas para el paciente en la siguiente visita y el segundo podría ser irreversible. En el análisis se incluyen los dos para poder compararlos con la bibliografía. El índice Kappa ponderado cuadrático en nuestro caso fue 0,876 lo que supone según la tabla propuesta por Landis y Koch un grado de acuerdo «muy bueno» (24). En todo caso aunque, se utilizase el método de asignación de pesos lineal, el resultado seguiría siendo parecido.

Las discrepancias producidas además de escasas no tienen trascendencia clínica, ya que el $92,3 \%$ fueron tan solo en un grado en la clasificación del ETDRS y en los dos casos $(7,7 \%)$ que fueron de dos grados no afectaba a la decisión de tratamiento ya que fueron entre no retinopatía y moderada. Las discrepancias nunca fueron superiores a dos escalones.

Aunque no se encontraron RD proliferativas, no consideramos que sea un inconveniente para la validación de la técnica, ya que el objetivo es el cribado de lesiones en pacientes diabéticos tipo 2 del ámbito comunitario y no hospitalario, en los que el número de lesiones graves sin diagnosticar y tratar es escaso (26). 
Llama la atención que los problemas de transparencia de medios son un $12 \%$ más frecuentes en los ojos discordantes (diferencia no significativa probablemente por el pequeño tamaño de la muestra de estos), por lo que cabe pensar que una vez tratados estos problemas la concordancia aún sería mejor.

La biomicroscopía leída por un solo oftalmólogo es un instrumento fiable como patrón de referencia para el diagnóstico de la retinopatía diabética.

\section{BIBLIOGRAFÍA}

1. Tamayo-Marco B, Faure-Nogueras E, Roche-Asensio MJ, Rubio-Calvo E, Sánchez-Olivan E, Salvador JA. Prevalence of diabetes and impaired glucose tolerance in Aragon, Spain. Diabetes Care 1997; 20: 534-536.

2. Castell $C$, Tresseras $R$, Serra J, Goday A, Lloveras G, Salleras L. Prevalence of diabetes en Catalonia (Spain): an oral glucose tolerance test-based population study. Diabetes Res Clin Pract 1999; 43: 33-40.

3. Muniz J, Hervada J, Juane R, Lopez-Rodriguez I, CastroBeiras A. Prevalence of diabetes mellitus in the population aged 40-69 years in Galicia, northwest Spain. Diabetes Res Clin Pract 1995; 30: 137-142.

4. Hove MN, Kristensen JK, Lauritzen T, Bek T. The prevalence of retinopathy in an unselected population of type 2 diabetes patients from Arhus County, Denmark. Acta Ophthalmol Scand 2004; 82: 443-448.

5. MacCuish AC. Early detection and screening for diabetic retinopathy. Eye 1993; 7: 254-259.

6. Leske MC, Wu SY, Nemesure B, Hennis A; Barbados Eye Studies Group. Risk factors for incident nuclear opacities. Ophthalmology 2002; 109: 1303-1308.

7. Moss SE, Klein R, Klein BE. The incidence of vision loss in a diabetic population Ophthalmol 1988; 95: 1340 1348.

8. Fong DS, Aiello L, Gardner TW, King GL, Blankenship G, Cavallerano JD, et al. Retinopathy in diabetes. Diabetes Care 2004; 27: S84-S87.

9. Vijan S, Hofer TP, Hayward RA. Cost-utility analysis of screening intervals for diabetic retinopathy in patients with type 2 diabetes mellitus. JAMA 2000; 283: 889-896.

10. Scanlon PH, Malhotra R, Greenwood RH, Aldington SJ, Foy $C$, Flatman $M$, et al. Comparison of two reference standards in validating two field mydriatic digital photography as a method of screening for diabetic retinopathy. Br J Ophthalmol 2003; 87: 1258-1263.

11. Stellingwerf C, Hardus PL, Hooymans JM. Two-field photography can identify patiens with vision-threatening dia- betic retinopathy: a screening approach in the primary care setting. Diabetes Care 2001; 24: 2086-2090.

12. Baeza Díaz, M, Gil Guillén V, Orozco Beltrán D, Pedrera Carbonell V, Ribera Montes C, Pérez. Pons I, et al. Validez de la cámara no midriática en el cribado de la retinopatía diabética y análisis de indicadores de riesgo de la retinopatía. Arch Soc Esp Oftalmol 2004; 79: 433-441.

13. Scanlon PH, Malhotra R, Thomas G, Foy $C$, Kirkpatrick $J N$, Lewis-Barned $N$, et al. The effectiveness of screening for diabetic retinopathy by digital imaging photography and technician ophthalmoscopy. Diabet Med 2003; 20 : 467-474.

14. Liesenfeld B, Kohner E, Piehlmeier W, Kluthe S, Aldington $S$, Porta $M$, et al. A telemedical approach to the screening of diabetic retinopathy: digital fundus photography. Diabetes Care 2000; 23: 345-348.

15. Harding SP, Broadbent DM, Neoh C, White MC, Vora J. Sensitivity and specificity of photography and direct ophthalmoscopy in screening for sight threatening eye disease: the Liverpool Diabetic Eye Study. BMJ 1995; 311: 1131-1135.

16. Herbert HM, Jordan K, Flanagan DW. Is screening with digital imaging using one retinal view adequate? Eye 2003; 17: 497-500.

17. Olson JA, Strachan FM, Hipwell JH, Goatman KA, McHardy KC, Forrester JV, et al. A comparative evaluation of digital imaging, retinal photography and optometrist examination in screening for diabetic retinopathy. Diabet Med 2003; 20: 528-534.

18. Prasad S, Kamath GG, Jones K, Clearkin LG, Phillips RP. Effectiveness of optometrist screening for diabetic retinopathy using slit-lamp biomicroscopy. Eye 2001; 15: 595601.

19. Argimón JM, Jiménez J. Métodos de investigación clínica y epidemiológica. Barcelona: Harcourt; 1999.

20. Silva Ayçaguer LC. Muestreo para la investigación en ciencias de la salud. Madrid: Díaz de Santos; 1993.

21. Indications for photocoagulation treatment of diabetic retinopathy: Diabetic Retinopathy Study Report no.14. The Diabetic Retinopathy Study Research Group. Int Ophtalmol Clin 1987; 27: 239-253.

22. Cohen J. Weighted Kappa: Nominal scale agreement with provision for scaled disagreement or parcial credit. Psychological Bulletin 1968; 70: 213-220.

23. Altman DG. Practical Statistics for Medical Research. London: Chapman \& Hall; 1991.

24. Landis JR, Koch GG. The measurement of observer agreement for cetegorical data. Biometrics 1977; 33: 159-174.

25. Doménech JM. Fundamentos de diseño y estadística. UD 14: Medida del cambio: Análisis de diseños con medidas intrasujeto. Barcelona: Signo; 2003.

26. Delgado M. Discordancias entre los estudios de ámbitos hospitalario y comunitario cuando evalúan la misma pregunta de investigación. Gac Sanit 2002; 16:344-353. 